\title{
Fusobacterium necrophorum
}

National Cancer Institute

\section{Source}

National Cancer Institute. Fusobacterium necrophorum. NCI Thesaurus. Code C86401.

A species of anaerobic, Gram-negative, rod shaped bacteria in the phylum Fusobacteria.

This species is non-spore forming, does not hydrolyze dextran and is superoxide dismutase and lysine decarboxylase negative. F. necrophorum is found in the intestinal tract of humans and animals, but is more likely to be associated with infections in animals. 